Results We studied 20 treatment resistant CC, 18 stable mild/ moderate A and $20 \mathrm{HC}$ subjects, matched for gender (M:F 10:10, 9:9,10:10, respectively), age [mean ( \pm SD) 57.1 years ( \pm 15.7 ), 51.7 years $( \pm 13.5), 58.8$ years $( \pm 13.5) ; \mathrm{p}=0.17]$, and lung function [3.02L ( \pm 0.98$), 2.96 \mathrm{~L}( \pm 1.10), 3.20 \mathrm{~L}( \pm 0.99) ; p=0.72]$. CC had significantly greater UTC intensity $(p=0.006$, see Abstract S116 Figure 1) and cough responses $(p=0.002)$ compared to HC. Females had significantly greater UTC $(p=0.001)$ and cough responses $(p<0.001)$ compared to males. There was no significant difference in UTC intensity ( $p=0.449$ ) or cough responses $(p=0.997)$ between A and HC. Conclusions In a randomised double-blind capsaicin cough challenge:

- Chronic cough patients perceive a more intense UTC sensation and also demonstrate a greater magnitude of cough response than healthy controls.

- Females perceive a more intense UTC sensation than males. These findings suggest both a heightened sensory experience in addition to an excessive motor response in patients with chronic cough. This data could be explained by sensitisation of afferent pathways (ie, peripheral and/or central sensitisation) but also by impaired inhibitory control mechanisms.

\section{S117 4 H COUGH FREQUENCY MONITORING WITH THE LEICESTER COUGH MONITOR}

doi:10.1136/thx.2010.150946.18

${ }^{1} \mathrm{~K}$ K Lee, ${ }^{1} \mathrm{~A}$ Savani, ${ }^{1} \mathrm{~S}$ Matos, ${ }^{1} \mathrm{C}$ Woods, ${ }^{2} \mathrm{D}$ Pavord, ${ }^{1} \mathrm{~S}$ S Birring. ${ }^{1}$ King's College Hospital, London, UK; ${ }^{2}$ Glenfield Hospital, Leicester, UK

Introduction The Leicester Cough Monitor (LCM) is a validated 24-h automated cough frequency monitor. Shorter recordings are more convenient for patients and take less time to analyse but their validity for assessing cough is not known. We assessed the relationship between short duration and 24-h recordings.

Methods 100 patients (57 females) with chronic cough underwent 24-h ambulatory cough frequency monitoring with the LCM. Patients completed diaries to identify awake and sleep periods. Cough frequency was determined by automated analysis and presented as 24 -h cough frequency $\left(\mathrm{CF}_{24}\right)$, awake cough frequency, sleep cough frequency, and short duration (1-6h). Subjective cough severity was assessed by cough visual analogue scale and

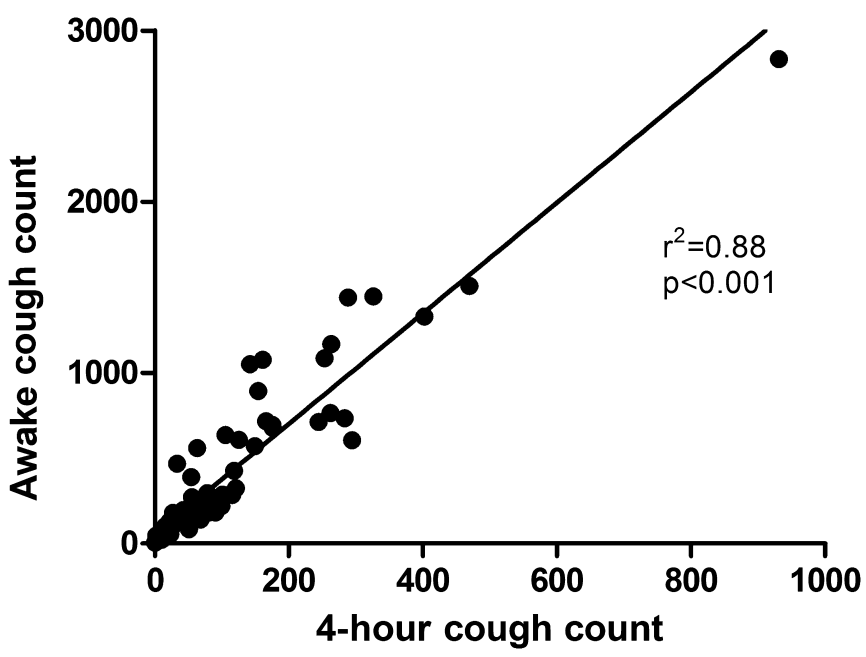

Abstract S117 Figure 1 Relationship between 4-h cough counts and awake cough counts. quality of life questionnaire (Leicester Cough Questionnaire, LCQ). The optimal short cough recording duration was determined by assessing its relationship with awake cough frequency and subjective cough severity. The responsiveness of short duration recordings was tested in 20 patients undergoing trials of therapy.

Results The median (IOR) 24-h cough frequency was 11.5 (5.8 to 26.6) coughs/h, awake cough frequency 13.2 (7.6 to 37.5) coughs/h and sleep cough frequency 4.2 (1.0 to 9.2) coughs/h. 4-h cough counts correlated strongly with both awake and 24-h cough counts; $r^{2}=0.88$ and $r^{2}=0.87$ respectively (Abstract S117 Figure 1 ). There was a moderate relationship between 4 -h cough frequency $\left(\mathrm{CF}_{4}\right)$ and LCQ and cough VAS $(r=-0.48, p<0.001$ and $0.49, p<0.001)$ which was comparable to that between awake cough frequency and LCO and cough VAS. The effect size of change in $\mathrm{CF}_{4}$ after therapeutic trials was 0.55 .

Conclusions Shorter duration cough frequency recordings with the LCM accurately reflect daytime and 24-h cough frequency in patients with chronic cough. They can be used to assess daytime cough frequency and the response to trials of therapy.

\section{S118 ACUTE HEMISPHERIC STROKE PATIENTS HAVE REDUCED FUNCTIONAL RESIDUAL CAPACITY AND COUGH FLOW RATES}

doi:10.1136/thx.2010.150946.19

P R Rao, K W Ward, C R Reilly, G R Rafferty, J Moxham. King's College London, London, UK

Introduction Cough is a complex manoeuvre, requiring coordinated action of the respiratory and upperairway musculature. The mechanisms of impaired cough following hemispheric stroke are unclear. Reduced functional residual capacity (FRC) may impair cough due to the effect of lung volume on the length and pressure generating capacity of the expiratory muscles. We compared FRC (primary outcome) and peak cough flowrate for voluntary cough (PCFR, secondary outcome) in stroke patients and healthy controls.

Methods 27patients and 30 healthy controls were studied. Stroke patients were within 2 weeks of first-ever middle cerebral artery infarct. Stroke severity was scored by a clinician (NIHSS score, worst=31). FRC was measured by helium dilution using a dry rolling seal spirometre. To measure PCFR, subjects wore a tightfitting facemask and were asked to cough forcefully into the spirometre. During these measurements, the volume inspired before the cough manoeuvre was also recorded. Measurements were performed in a chair with the back reclined to $45^{\circ}$, mimicking patient position in hospital. FRC and PCFR data were expressed as $\%$ predicted. $^{1}$

Results Patients' median NIHSS score was 4 (IOR 2-6) reflecting mild disability. FRC \% predicted, the volume inspired before cough and PCFR were significantly reduced in patients. Both FRC and the volume inspired before cough were significant predictors of PCFR.

Conclusions FRC (\% predicted), the volume inspired before cough and PCFR were significantly reduced in acute hemispheric stroke patients. Higher peak cough flow rates are associated with greater lung volume prior to cough. Interventions that increase FRC, for example, continuous positive airway pressure and upright sitting may improve cough function in stroke patients. 